\title{
Design and Implementation of Decentralized Swarm Intelligence E-Commerce Model Based on Regional Chain and Edge Computing
}

\author{
Xiaofang Luo \\ Education Supervision Office, Yiwu Industrial and Commercial College, Yiwu 322000, China \\ Correspondence should be addressed to Xiaofang Luo; 1xf1999010031@ywicc.edu.cn
}

Received 8 February 2021; Revised 17 April 2021; Accepted 28 April 2021; Published 5 May 2021

Academic Editor: Wei Wang

Copyright (c) 2021 Xiaofang Luo. This is an open access article distributed under the Creative Commons Attribution License, which permits unrestricted use, distribution, and reproduction in any medium, provided the original work is properly cited.

\begin{abstract}
In this paper, we investigate the model of decentralized groupwise e-commerce through regional chains combined with edge computing algorithms and design and implement it. A blockchain-based architecture for remote sensing resource and service transactions is proposed. The architecture utilizes the characteristics of nontamperable and traceable data on the blockchain to solve the problem of mutual distrust between the transaction parties, uses MongoDB for data retrieval and filtering to solve the problem that it is difficult to query flexibly on the blockchain, uses the file platform to save public resources to solve the problem that it is very expensive or even infeasible to store images or large texts in the blockchain, and ensures remote sensing resources and services related to the effective storage of images and description texts. The Ethernet features are described and analyzed to assess their suitability as a technical basis for the corresponding solutions. To assess the ability of the Ethernet-based system to solve solvable problems, its practical use cases are analyzed. The results of the analysis show that five main issues need to be addressed when applying Ethernet to e-commerce, namely, low scalability, lack of distributed storage, lack of convenience, reliability, and functional integrity. To exploit the idea of using blockchain technology to solve complex problems and to overcome the challenges identified in the existing Ethernet-based implementation solutions, an optimization idea is proposed by creating a multiblockchain architecture based multimodule solution in the Ethernet framework. Finally, the application prospects of the proposed information system are discussed.
\end{abstract}

\section{Introduction}

Although the Internet has brought such a great change, due to the technical characteristics of the Internet, there are still some shortcomings in the Internet-based business model, mainly including the high cost of credit acquisition and central platform monopoly [1]. Credit is the basis of transactions, and on the Internet, it is usually necessary to introduce a trusted third party as a guarantee before a transaction can be concluded, which increases the cost for SMEs. Also, there are obvious drawbacks of centralized e-commerce platforms. On the one hand, since the transactions involve two or more countries if the platform deposits a large amount of import and export data of other countries, it may affect the import and export trade of that country, which is not tolerated by any country; on the other hand, centralized platforms may act against the overall interests of participants because of the pursuit of excessive profits, such as hegemonic terms or hidden terms.

While many companies in the e-commerce space expect to use blockchain for a variety of purposes, there are still some problems with its actual use by these companies [2]. These problems are based on 3 phenomena that exist at the intersection of blockchain technology and e-commerce. These issues are of great research interest and form the main theoretical and practical issues of this paper. The first phenomenon is the large number of projects based on this technology, while there is very little data about its use in e-commerce. The second is the uncertainty of the common ground between blockchain and the business sector. The third is the lack of practical experience with the use of this technology on a large scale [3]. There is not enough knowledge about specific blockchain projects applied to the e-commerce sector. There is some uncertainty about the 
theory related to the relationship between the functionality provided by blockchain and e-commerce, and most papers lack knowledge based on a holistic understanding. There are problems in the process of introducing blockchain in e-commerce; however, the theoretical basis of the current solution is insufficient. The problem of optimal choice in developing solutions arises. The point of contact between blockchain mechanisms and e-commerce problems has not been specified. There is the problem of optimization of existing blockchain-based solutions until their large-scale application.

A blockchain-based electronic data storage system is designed, which makes full use of the decentralization and immutability of blockchain to anchor the key data information on the main chain and control the access rights of different users to the electronic data, effectively solving the security problems of electronic data storage. It provides users with services, such as data upload, download, query, comparison, and authorization, and introduces distributed storage technology and user point mechanism to increase the reliability of the system. This paper combs through the literature on e-commerce platform business model and the impact of blockchain technology on e-commerce business model and finds that most of the current studies focus on the innovation of e-commerce platform business model based on the Internet, but there are few studies on the business model of blockchain-based e-commerce platform, and there is a lack of studies that use case studies to analyze the business model of blockchain-based e-commerce platform. This paper analyzes the characteristics and shortcomings of the existing e-commerce platform business model based on the "threedimensional model" theory and explores and analyzes the business model of blockchain-based e-commerce platform based on the existing research results of blockchain technology to build e-commerce platform with the case of silk chain. This paper analyses the current mainstream e-commerce import and export platform business model and points out its features and shortcomings [4]. Through the case study of Silk Chain, we focuse on the market position, business model, and economic sustainability of the e-commerce platform business model based on Silk Chain and argue whether Silk Chain can make up for the shortcomings of the traditional model and whether the Silk Chain model has the learnability. Finally, based on the conclusion of the research on silk chain and the current situation of the development of e-commerce business model, we put forward relevant countermeasures and suggestions. This paper contributes to the theoretical development of blockchain technology applied to e-commerce issues and puts forward conclusions and suggestions with a practical value. The main research issues include the blockchain diversity, applicability, and challenges of practical application in e-commerce. The e-commerce issues identified in this paper are described as a whole, providing a relatively broad analysis and opinion. This paper explores the complete unfolding path of thought from the blockchain itself to the applications used to solve e-commerce problems.

\section{Related Studies}

Ale et al. [5] compare the impact of distance between buyers and sellers on eBay's online cross-border transactions and offline international trade traffic. Using the same 62 countries and the same set of goods for both online and offline transactions, they find that the impact of distance is $65 \%$ smaller when trading through the eBay online platform than when trading offline. Ren et al. examined the drivers and barriers to cross-border e-commerce in the EU [6]. By analyzing data from an online consumer survey panel, they found that online cross-border trade of goods in linguistically dispersed EU markets has significantly lower distancerelated transaction costs compared to offline transactions for similar goods, but that language-related trade costs increase and a $1 \%$ increase in the use of efficient and flexible crossborder payment systems increases cross-border e-commerce by $7 \%$, etc. Atzori et al. analyze the impact of the EU general data protection regulation (GDPR) on blockchain technology and investigate whether blockchain can meet the requirements of the GDPR [7]. Kherraf et al. studied the application of blockchain in ballots and found that the use of blockchain makes ballots simpler because the process and results of ballots do not require a centralized body to pass through based on the consensus mechanism of blockchain, and each participant can vote and verify the votes by themselves [8].

Use blockchain to solve the supply chain, cross-border payment, and logistics problems of cross-border e-commerce houses [9]. Aral studied the impact of blockchain technology on traditional cross-border remittance payments and concluded that swifts will still dominate the crossborder payment industry in the short term, but emerging technologies such as Ripple will transform the remittance industry in the long term [10]. Moura and Hutchison analyze the challenges and blockchain technology features in global supply chain and trade operations and consider the potential of blockchain technology applications in the supply chain and coordination industry [11]. Shen analyzed the pain points of financial institutions in carrying out supply chain finance business and found that they face challenges in credit identification, transaction supervision, risk control, and other aspects of supply chain finance business, based on which they analyzed the application of blockchain technology in supply chain finance business and found that blockchain technology can provide new ideas to solve some of the problems [12]. Some scholars analyzed the problems of the cross-border e-commerce payment model, combined with the characteristics of blockchain technology, and discussed the role and challenges of using blockchain technology to optimize cross-border e-commerce payment model [13].

In summary, the basic concepts, characteristics, and applications of blockchain technology are the key research areas, among which the research in the literature review category has started to increase since 2018, and the research on the application of technology to specific scenarios is also increasing, but it is biased toward theoretical research; in the foreign literature, the blockchain research has a broader 
scope, including the applied research in social and economic activities in addition to finance and also foreign research on specific There are relatively more case studies on specific blockchain applications abroad. The feasibility of applying blockchain technology to the e-commerce industry, the use of blockchain technology to solve the problems of the e-commerce industry, and the construction of a new platform or ecology show that the impact of blockchain technology on the e-commerce industry has received the attention of the academic community. The foreign literature mainly focuses on supply chain management and functional contracts, and there is relatively little literature on the impact on e-commerce business models.

\section{Regional Chain Fusion Edge Computing for E-Commerce Model Design}

3.1. Analysis of Regional Chain Convergence Edge Computing. The division of application services is very important for designing a self-adaptive, economical, and efficient scheduling system for edge computing, where the modeling of application services and the design of scheduling policies are the most critical aspects [14]. Since the scheduling strategy of microservices is based on the model of the scheduling system, considering the heterogeneity of computing and network resources existing in edge computing, past studies have simplified the model of the system as much as possible to reduce the difficulty of the study and thus cannot be well applied to the scenario where a large number of edge computing nodes are available for schedule exist. First, we specifically analyze the shortcomings in the past studies, introduce the process and key issues of application partitioning and microservice scheduling, and propose a new edge computing application scheduling model and application partitioning algorithm.

The current research on application partitioning and scheduling is mainly based on the cloud computing scenario. When different microservices are scheduled by the partitioning algorithm and assigned to the same cloud center, by default there is no communication cost between them, and all communication time cost comes from the cross-end communication cost when scheduling to different clouds [15]. However, in the edge computing scenario, microservices may be scheduled on each clock computing resource at the edge, and due to the heterogeneity of network resources, the communication time cost between microservices cannot be neglected, and there may also be not symmetric cross-end communication cost, so the simple research model in the past does not apply to the microservice architecture scenario of edge computing. Therefore, this section proposes a new microservice scheduling model for edge computing scenarios. The model addresses the shortcomings of previous studies by removing two premises and considering the computation and bandwidth resources of different cloud heterogeneous types, which can well generalize the various conditions of edge computing and cloud computing when performing service scheduling. Each node has two computational weights that represent the computational time cost of this microservice in the local edge cloud and scheduling to offsite servers, respectively:

$$
w_{i}^{2}=\left\langle w_{i}^{2 L}, w_{i}^{O}\right\rangle .
$$

$w_{i}^{2}$ is the ratio of the execution speed of the scheduled server to the execution speed of the local edge cloud server (or the inverse of its average task completion time). Usually, the computing power of the offsite server $w_{i}^{O}$ is higher than that of the local edge device. Therefore, the total execution time cost of microservice computing can be obtained expressed as:

$$
T_{\text {comp }}(I)=\sum_{v_{i} \in V} I_{i}^{2} \times w_{i}^{2 L}+\sum_{v_{i} \in V}\left(I_{i}^{2}-1\right) I_{i}^{2} \times w_{i}^{L} .
$$

Also, the scheduling time of microservices between the local edge cloud and offsite servers is $T_{\text {comp }}(I)$, where $v_{i} \in V$ is the amount of data required to schedule a microservice and $L$ is the transmission bandwidth, and the total transmission time cost of microservice scheduling is

$$
T_{\text {total }}(I)=\sum_{v_{i} \in V} I_{i}^{-2 i} \times w_{i}^{-2 r L} .
$$

Since the applications were simply divided into edge and central cloud in the past studies, the communication and transmission between them are not considered if the services are all divided in the central cloud. However, in the real scenario, the divided services are dispatched not only to the central cloud but also to other offsite edge computing servers, and the communication and transmission costs between them cannot be ignored. Second, the communication cost of microservices at different ends is not symmetric, i.e., the communication cost of microservices between local edges and offsite clouds is not equal. Because, in reality, different clouds have different network conditions, the communication transmission between microservices and computing services is related to the link transmission rate and network bandwidth blocking between them:

$$
w\left(e\left(v_{i}, v_{j}\right)\right)=\frac{\mathrm{in}_{i j}^{2}}{B_{\text {upload }}}+\frac{\text { out }_{i j}^{2}}{B_{\text {download }}} .
$$

For simplicity, this section $w_{i j}^{2}$ expresses all possible communication time costs between microservices in the following equation:

$$
w_{i j}^{2}=\left\langle w_{i j}^{2 L}, w_{i j}^{O}\right\rangle .
$$

After the execution location of the microservice is determined, the values of these costs are also determined. Thus, the total communication time cost between microservices is

$$
T_{\text {comm }}(I)=\sum_{i . j}\left(w_{i j}^{2 L} I_{i}^{-2 i} \times w_{i}^{-2 r L} w_{i j}^{O}\left(I_{i}^{2}-1\right)\right) .
$$

Partitioning algorithms should adapt to changes in networks and devices. For example, the optimal partitioning $T_{\text {comm }}(I)$ for high-bandwidth, low-latency networks, and low-volume client clusters may not be appropriate for highvolume client clusters with poor network connectivity. Since 
network conditions can only be measured at runtime, the partitioning algorithm should be a real-time, online process:

$$
T_{\text {total }}(I)=T_{\text {comm }}(I)+T_{\text {comp }}(I) .
$$

Some application components are flexible microservices that can be processed locally on a mobile device's processor or a remote cloud server or a nearby edge server $I_{i}^{-2 i}$. Many services fall into this category, and scheduling decisions depend on whether the cost of communication is greater than the difference between local and remote costs. Assume that the various services have been annotated by the programmer with their types [16]. For nonschedulable microservices, a nonschedulable decision must be made. However, for schedulable tasks, since scheduling all application tasks to a cloud/edge server is not necessary or efficient in any case, one must consider which tasks should be executed locally on the mobile device and which tasks should be executed on the mobile device. While scheduling to a server for remote execution, this decision is made based on the available network, network response time of the mobile device relative to the remote server, or energy consumption. The mobile device must make an offload decision based on the results of the dynamic optimization problem. A division of the edge computing scheduling model is shown in Figure 1.

For the blockchain-based remote sensing resources and services trading platform, the transaction information of platform users, such as remote sensing resources and services description information, bidding information, remote sensing resources and services bidding results, transaction arbitration results, and other information need to be stored differently according to their importance. Put all remote sensing resources and services transaction logic and core data in the blockchain. While guaranteeing the trustworthiness of the transaction, the platform needs to ensure flexible queries of information related to remote sensing resources and services and solve the storage capacity limitation of the blockchain.

A dynamic credibility-based improved ant colony algorithm, computation-based ACO (RACO), is proposed for grid task scheduling. RACO introduces dynamic adjustment factors of spatial and temporal efficiency and adopts both local and global pheromone update strategies. To solve the problem that the standard BP neural network converges slowly and easily falls into local minima, which increases the network error, a variable step length BP network prediction algorithm with the introduction of momentum term is proposed. The improved BP network algorithm is applied to prediction by changing the step size of the learning rate based on the introduction of momentum term factor (JPCO), and the error performance curves of the traditional $\mathrm{BP}$ algorithm and the improved BP algorithm are obtained through simulation experiments. The simulation validation shows that the improved BP neural network effectively speeds up the convergence speed and makes the network error avoid falling into local minima, and the prediction effect is better.
If more query features are added to a smart contract, more arrays, maps, or other data structures need to be introduced, resulting in more complex contract code. More complex contracts mean not only more gas to deploy the contract but also more error-prone [17]. However, errors cannot be corrected after the contracts are deployed, resulting in high maintenance costs later on. The participants are divided into two parts: cross-border trade participants and blockchain technology implementation participants. In the first part, for cross-border trade participants, besides government agencies, they mainly include producers in exporting countries, traders in exporting countries, third-party service providers in exporting countries, Internet-based cross-border e-commerce platforms, importers in importing countries, consumers in importing countries, and third-party service providers in importing countries. Among them, the reasons for Internet-based cross-border e-commerce platforms to become participants will be analyzed specifically in the following. In the second part, for the blockchain system implementation participants, they also need to include the representative platform management, technology development, and maintenance personnel and miners. Among them, the platform management is similar to the management in a company, which handles platform-related matters in daily life, such as issuing technical development tasks. The technical development and maintenance personnel are mainly involved in the development and maintenance of the platform functions, and miners are mainly involved in the verification of transaction information in the blockchain system, thus enabling the blockchain system to realize the bookkeeping function, as shown in Figure 2.

The core of the operating system of the blockchain-based cross-border e-commerce platform lies in the ability to complete cross-border transactions without relying on any trusted third party. Due to the different number of participating nodes and the different services provided by the platform, it can theoretically contain a variety of services of B2B, B2C, and C2C. Since the realization logic of the three modes is the same, only B2B is taken as an example here. As shown in Figure 2, eight links are needed to complete B2B transactions on the cross-border e-commerce platform based on a blockchain platform [18]. First, the seller puts the information of goods and the buyer puts the information of monetary assets into the cross-border e-commerce trade chain for verification. Second, the seller puts the goods information on the Internet-based cross-border e-commerce platform for shelving. Third, the buyer purchases and places an order through the Internet-based cross-border e-commerce platform. Fourth, the Internet-based crossborder e-commerce platform sends the information containing order information, transaction process information, and other transaction information to the cross-border e-commerce trade chain. Fifth, the transaction information is written to the initialization block. Sixth, the block containing the transaction information is broadcast in the trade chain. Seventh, both buyers and sellers accept that the 
transaction has been completed. Eighth, the initialization block is connected to the previous block and the trade chain is extended by one block.

\subsection{Decentralized E-Commerce System Implementation} Design. E-commerce environment development, as a driving factor that can promote business model innovation, is manifested in the operation of the business model system as a technical path that can achieve the purpose. Also, different innovation paths formed by different combinations of application depth, methods, and means have different influencing factors on business model innovation [19]. Based on the above research, this paper proposes 6 innovation paths of business model innovation for commercial enterprises and 7 influencing factors derived from the analysis and refinement. The six innovation paths mentioned above all have the influencing factor of satisfying customer value proposition, so when selecting the innovation paths through the influencing factors, we can consider not to study the influencing factor of satisfying customer innovation proposition.

Based on the above research on the influencing factors and technological innovation paths, this paper researches the selection of business model innovation paths based on the e-commerce environment through qualitative analysis and also selects multiple innovation paths by the role played by different influencing factors through the selection matrix. In practice, commercial enterprises can consider the multidimensional color scale selection matrix as a screening mold according to the development of the e-commerce environment and combining with their situation, and through such a multidimensional color scale selection matrix, it can select relatively suitable business model innovation paths for business model innovation under different circumstances [20]. Without considering which path affects the business model innovation through which factors, the factors are overlapped in the form of color chips, and the more the layers are overlapped, the darker the color is, and the color scale is formed from light to dark, which means that the darker the color is, the more attributes the corresponding innovation path affect the business model innovation and the advantages it brings to the business model will be. The darker the color is, the more attributes the corresponding innovation path has on the business model innovation and the more advantages it can bring to the business model. Then, the business enterprise can determine which color scale the innovation path corresponds to according to its own needs for the relevant attributes and further correspond the alternative paths presenting different influencing factors to the color scale to choose the suitable innovation path for the enterprise.

Blockchain-based cross-border e-commerce platforms have three major features: polycentricity, low cost of trust acquisition, and high openness. The centralized value of blockchain is that the blockchain is not controlled by any single node. The traditional cross-border e-commerce platform is controlled by a single company, so the traditional cross-border e-commerce platform is centralized, while the blockchain-based cross-border e-commerce platform is noncentralized. If the blockchain platform is built with public chain technology, it is decentralized, and if the platform is built with alliance chain technology, it is polycentric.

For a cross-border transaction, on the Internet crossborder e-commerce platform, the way the two sides of the transaction obtain each other's trust relies on the third-party trusted body, the seller obtains the platform's audit, the consumer needs to pay the payment and commission to the platform in advance, and the platform guarantee in this model plays a role in reducing the cost of trust acquisition; on the blockchain cross-border e-commerce platform, trust comes from the consensus protocol of the blockchain platform; the consensus protocol is a core of the blockchain system, which enables all nodes to synchronize data and allows the blockchain system to form an autonomous ecology, as it contains many provisions, such as data communication and transmission; the blockchain system can be updated, and the blockchain system is not controlled by any single node. Comparatively, the cost of acquiring trust is lower because transactions on the blockchain platform do not require the intervention of any trusted third party.

The blockchain-based cross-border e-commerce platform has better openness. At the technical level, the public chain system is open source, which means more applications will be developed and shared, such as smart contracts, which provides greater growth for the platform. At the level of international trade friction, due to the decentralized nature of the blockchain-based cross-border e-commerce platform, it has been a global platform from its inception, and since the platform is no longer vested in any party, governments do not need to worry about other countries using the platform to their disadvantage [21-23]. At the community governance level, due to the consensus agreement, the blockchain-based cross-border e-commerce platform is also bound to be highly autonomous, so the above points show that the platform has a high degree of openness.

The physical architecture of this platform consists of three parts: web front-end, web server, and data storage. The first part is the web front-end, through which users can access Ethernet, IPFS, and NodeJS server; the second part is the Web server, which uses NodeJS as the back-end server to provide Web services and listen to user blockchain events; the third part is the data storage composed of Ethernet blockchain, IPFS, and MongoDB. The third part is the data storage composed of Ethernet blockchain, IPFS, and MongoDB, which stores transaction data and smart contracts; IPFS stores image data and related specific description text describing remote sensing resources and services, and MongoDB carries out off-chain data backup. The physical architecture design of this platform is shown in Figure 3.

A pass-through certificate here refers to a negotiable cryptographic virtual proof of interest-based on blockchain technology. When a user owns a pass, it is equivalent to having some kind of rights on the blockchain, which can represent the right to use a product or service or the ownership of some virtual assets. However, the passwords 


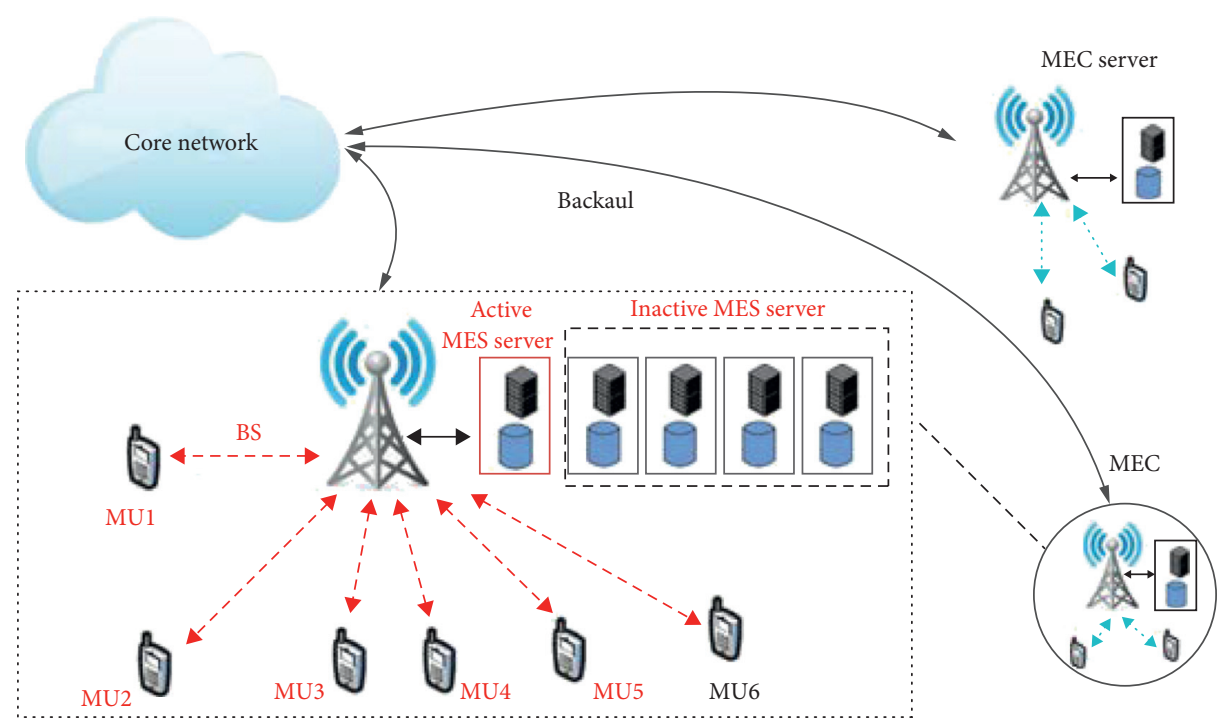

FIGURE 1: Division of edge computing scheduling model.

play not only its role of confirming rights for the blockchain platform but also its incentive role. In market economic activities, any rational person makes economic decisions based on making himself profitable, and the same is true in a blockchain-based cross-border e-commerce platform. By issuing passwords, it is possible to crowdfund the funds needed to build the platform, and by giving rewards to participants who contribute to the platform, it is possible to accelerate the development of the platform ecology, so the greater significance of the passwords system is that it provides incentives to enable the platform ecology to independently and efficiently carry out economic activities. However, the pass is not something necessary for the existence of a blockchain platform; just the platform of blockchain lacking pass cannot build an economic system around the pass, which will lead to the limitation of the application scenario based on the platform. On the contrary, by combining the blockchain system with the pass system, the potential of the business model of the blockchain-based cross-border e-commerce platform will be amplified to a greater extent.

\section{Results and Analysis}

4.1. Analysis of Simulation Results. The RACO algorithm takes the computing tasks generated by mobile devices and selects the offloading method based on the total cost consumption. The JPCO algorithm, on the other hand, not only allocates the channel reasonably to the user who needs to offload the task according to the priority but also improves the efficiency of the channel usage. Figure 4 shows the total cost consumption of the no offload calculation, RACO algorithm, and JPCO algorithm with the increasing number of mobile devices. We can see that there is a significant decrease in the total cost consumption using the JPCO algorithm over the local computation and RACO algorithms.

The average cost consumption of the RACO algorithm decreases by $50 \%$ based on local computation, and the average cost consumption of the JPCO algorithm decreases by $34 \%$ based on the RACO algorithm. And, from Figure 4, the time consumption of the RACO algorithm decreases more than $50 \%$ based on local computation, while the JPCO algorithm decreases only about $10 \%$ compared to the RACO algorithm. This is because we introduce a residual energy factor in the JPCO algorithm, which reduces the requirement for time delay when the residual energy is less. Therefore, the reduction in total time consumption is lower than the RACO algorithm, which uses time delay as an offloading criterion. As a measure of the energy-saving efficiency of the whole system, the JPCO algorithm decreases more than $50 \%$ overall than the RACO algorithm in terms of energy consumption, and the energy-saving becomes more and more effective as the number of computational tasks increases. From the above data analysis, we can obtain that the joint optimal computational offloading scheme proposed in this chapter has made great progress in reducing both time and energy consumption.

Figure 5 depicts the variation of the energy consumption of mobile devices and MEC servers in the cell with the number of deployed MEC servers, with the number of users set to 30. It can be seen from the figure that the energy consumption of the whole system is decreasing with the increase of servers. This is because as the number of servers increases, the computing resources of the cloud servers in the cell can meet the offloading requirements of more users, and the users can offload their computing tasks to the MEC servers when the wireless resources are satisfied. Also, the curve of the BFRO algorithm falls faster than ROA (random offloading algorithm) and consumes less energy. Before the number of servers can satisfy the offloading requirements of all users, the energy consumption of BERO decreases by less than $10 \%$ compared to $\mathrm{ROA}$, around $7 \%$. After reaching saturation, this figure reaches $21 \%$. The energy consumption of the JFLO algorithm decreases by more than $10 \%$ compared to BERO (at saturation). After the number of servers exceeds 7 , the decrease in energy consumption is less 


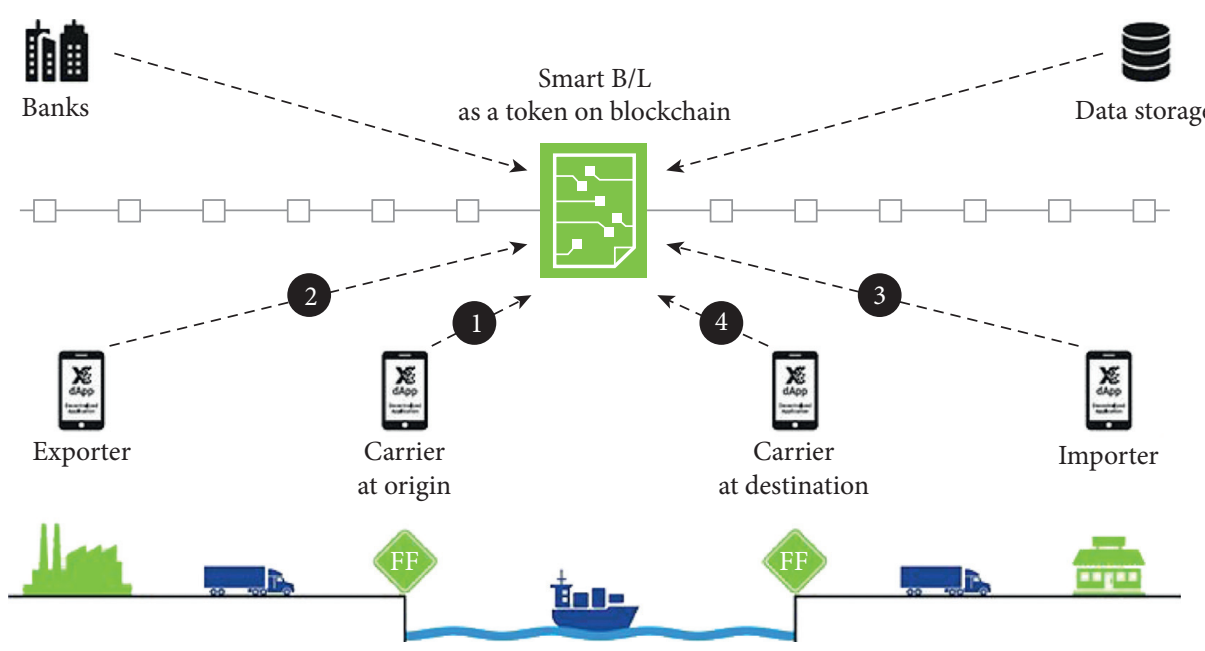

Figure 2: Blockchain-based B2B transaction path diagram for cross-border e-commerce.

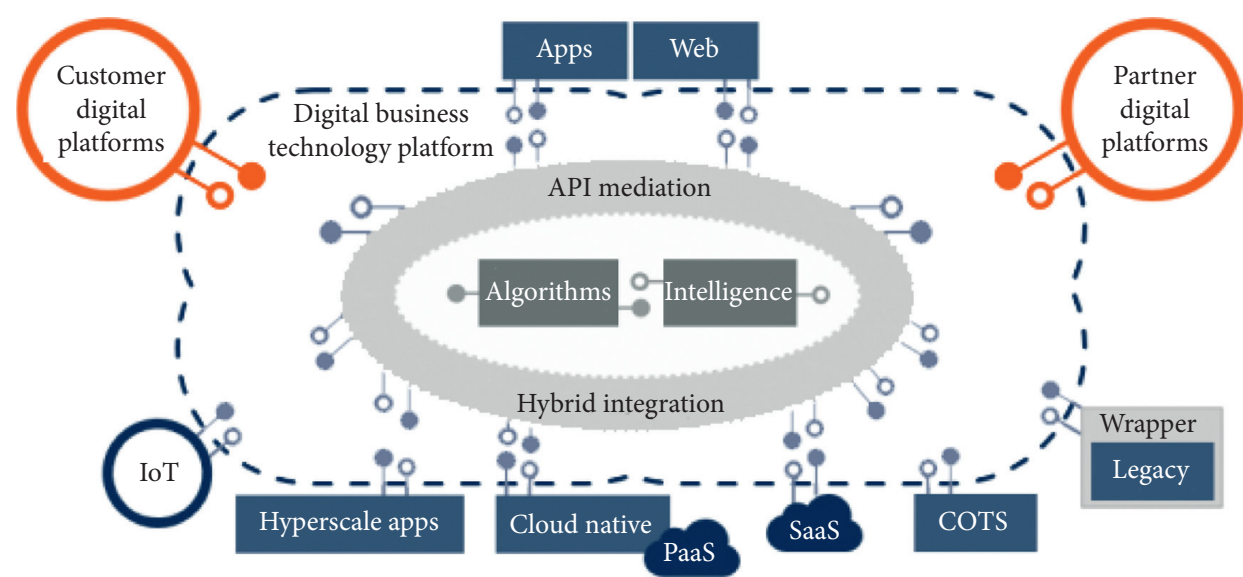

FIgURE 3: Physical architecture of the resource and service trading platform.

pronounced and leveled off. This is because the computational resources provided by the MEC servers can already satisfy the offloading requirements of the computational tasks, but the wireless resources are limited, so the energy consumption will not decrease endlessly. At this stage, some computing resources will be idle, and the next research goal is to increase the efficiency of MEC server usage.

This experiment compares and analyzes the performance of 5 scheduling methods by varying the input size of intermicroservice data for latency-sensitive application virtual reality game EFG tractor beam, configuring 5 auxiliary computing nodes for UAVs, one cloud center node, and one edge cloud node as schedulable nodes according to the table. The number of applications executed is still 3. Figure 6 shows that the average completion time of all 5 scheduling methods increases with the increasing input data of the application. When the application input data size is below $200 \mathrm{MB}$, the difference in completion time between these methods is not significant. However, as the input data increases, the completion time of these methods varies significantly from $750 \mathrm{MB}$ to $1050 \mathrm{MB}$. For the two scheduling methods, VMC and Greedy algorithm, although they can select the free and not too far away computing resources for scheduling when the number of applications is not too large, the algorithm is unable to select the computing nodes for scheduling due to the increasing amount of data transmission between application modules. When the input data size is $1180 \mathrm{MB}$, the computation time cost reduced by the service schedule is much smaller than the communication time cost brought by the transmission data, so the total completion time has been greatly improved, and the average completion time of these two algorithms has a high positive correlation with the data size.

For the scheduling method that is executed only locally, although the communication time cost due to the increase of input data does not have a particularly large impact on this method, it generates a lot of waiting time due to the lack of local computing resources so that the completion time of this scheduling model is always in a relatively high situation. For the MCMO algorithm proposed in this paper, since it fully considers the impact of system computation cost, communication, and waiting time cost, it dynamically adjusts the scheduling target according to different input data conditions so that the impact of the cost model is always kept 


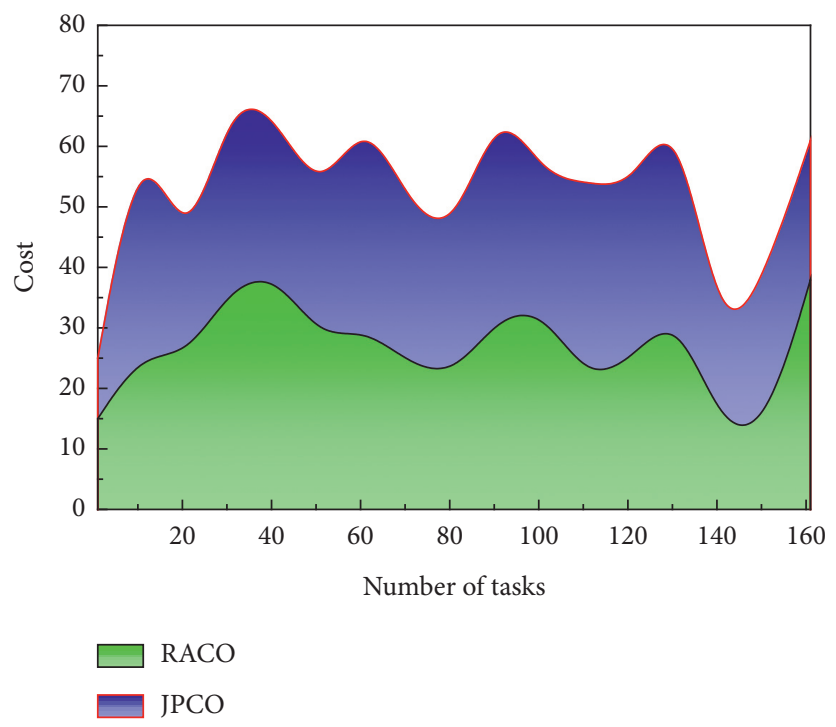

Figure 4: Total cost consumption of local calculation, RACO algorithm, and JPCO algorithm.

to a minimum. When the input data is $1050 \mathrm{MB}$, the average completion time of the MCMO algorithm saves $22.3 \%$ and $10.5 \%$ compared with the VMC algorithm and the greedy algorithm, respectively. However, when the size of the input data is above $1180 \mathrm{MB}$, the variability of several methods starts to gradually decrease again, which is because the input data is so large that the auxiliary computation can no longer be performed well with lightweight computing nodes such as drones, the available scheduling destinations are reduced, and with the increasing transmission time, the completion time of the predecessor tasks of microservices will keep increasing, which in turn increases the service waiting time.

4.2. System Performance Analysis. This article uses Hyperledger Caliper to perform testing of blockchain performance: a general-purpose framework that allows benchmarking different blockchain platforms, testing transactions from throughput, latency, and more. It allows users to test different blockchain solutions using predefined use cases and get a set of performance test results. The query returns all customer deposits and loan balances for a branch of the bank and involves joins between multiple tables. With multitable queries, the query results are aggregated, so all records are polled for each table, and it is not possible to rely on keywords to improve query efficiency; the main factor affecting the query statement is the amount of data transfer. It can be seen that Hadoop and Costately have a greater effect on query efficiency in this scenario. The main principle is that the shuffle phase of the Hive task reduces the data transfer time because the relevant files are distributed in the same node space. Under the 200 tps concurrent condition, the performance of each main method of remote sensing resource bidding based on a second-price sealed auction is good, with a $100 \%$ request success rate and throughput of
200 tps. The highest latency of the revealed method is 0.61 seconds. The average latency of all methods is 0.445 seconds. The resource consumption of the three test nodes is high, with node 1 reaching $178.2 \mathrm{MB}$, node 2 reaching $154.9 \mathrm{MB}$, and node 3 reaching $139 \mathrm{MB}$, as shown in Figure 7.

In the five tests, the performance results indicate the same $100 \%$ success rate of requests for each method. In the $100-300$ tps range, the average latency increases from 0.22 seconds to 0.595 seconds as the number of concurrent requests decreases, the maximum latency increases from 0.32 seconds to 0.83 seconds, and the platform throughput increases from $26 \mathrm{tps}$ to $231 \mathrm{tps}$. Therefore, the platform can meet real-life scenarios. Therefore, the platform can meet the high concurrent requests in real scenarios and can operate normally under the peak request scenario.

The query returns the names and deposit balances of all customers in a branch of the bank and involves two table associations. From the results in Figure 8, we can see that the HDFS original data allocation method has the lowest query efficiency, mainly, because the two files associated with the query are not in the same data node, resulting in the existence of partial data transfer. And, the query time of CoHadoop and Costately is the same, mainly, because CoHadoop uses the data partitioning of the join keyword, and the placement of files and data blocks is the same as the way of CovStrategy data file allocation. Meanwhile, we extract some time points during the query process to obtain the network traffic situation, which verifies the experimental conclusion.

Compare the customer query in the cloud environment with the search in the Internet scenario, establish the feature vector of the dataset table, and measure the vector distance based on the cosine similarity of the feature vector; use the $\mathrm{BEA}$ algorithm to aggregate the dataset table by establishing the dataset table correlation matrix and to carry out the 


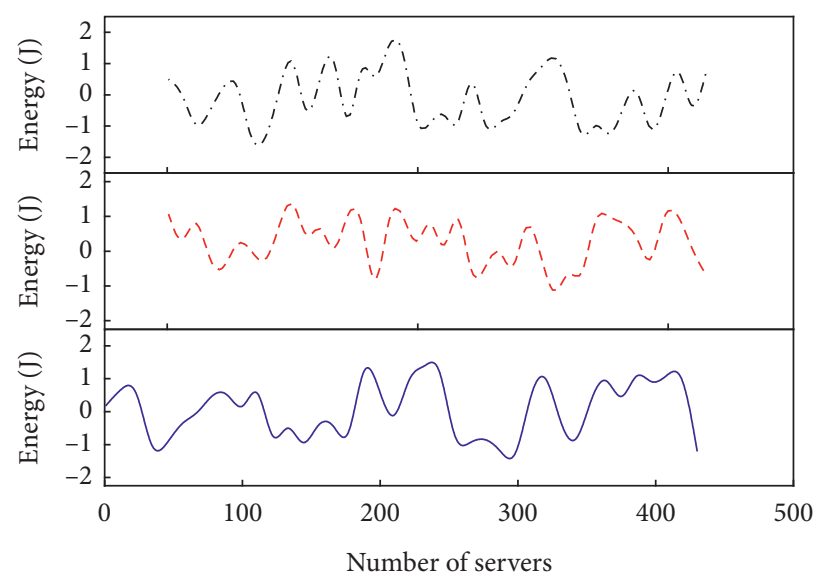

$$
\begin{aligned}
& \text {-.. ROA } \\
& \text {-- BFRO } \\
& \text { — JFLO }
\end{aligned}
$$

FIGURE 5: Graph of energy consumption with the number of MEC servers.

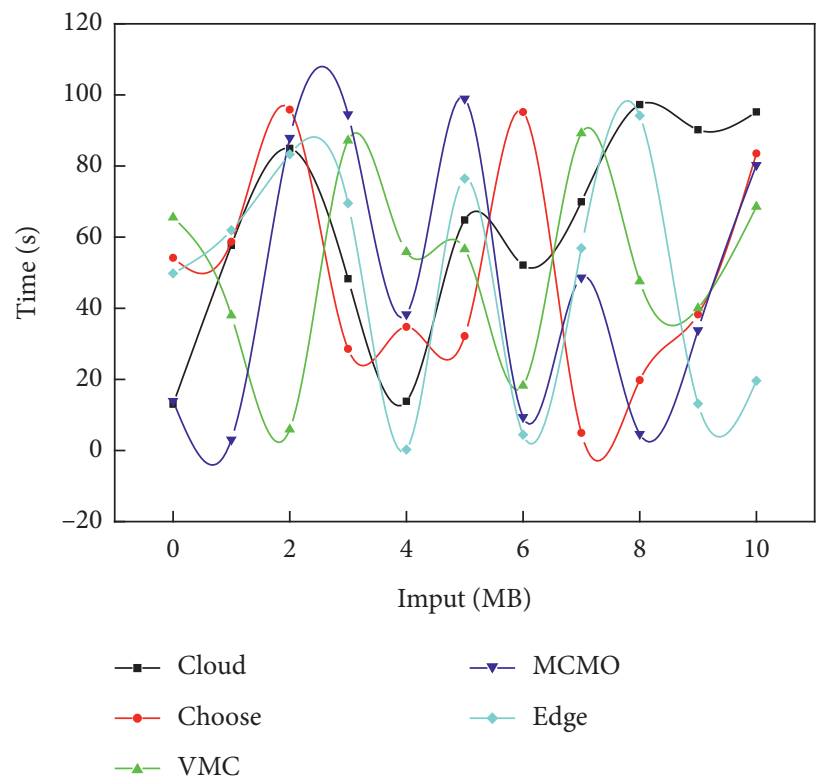

FigURE 6: Impact of application input size on computational scheduling. 


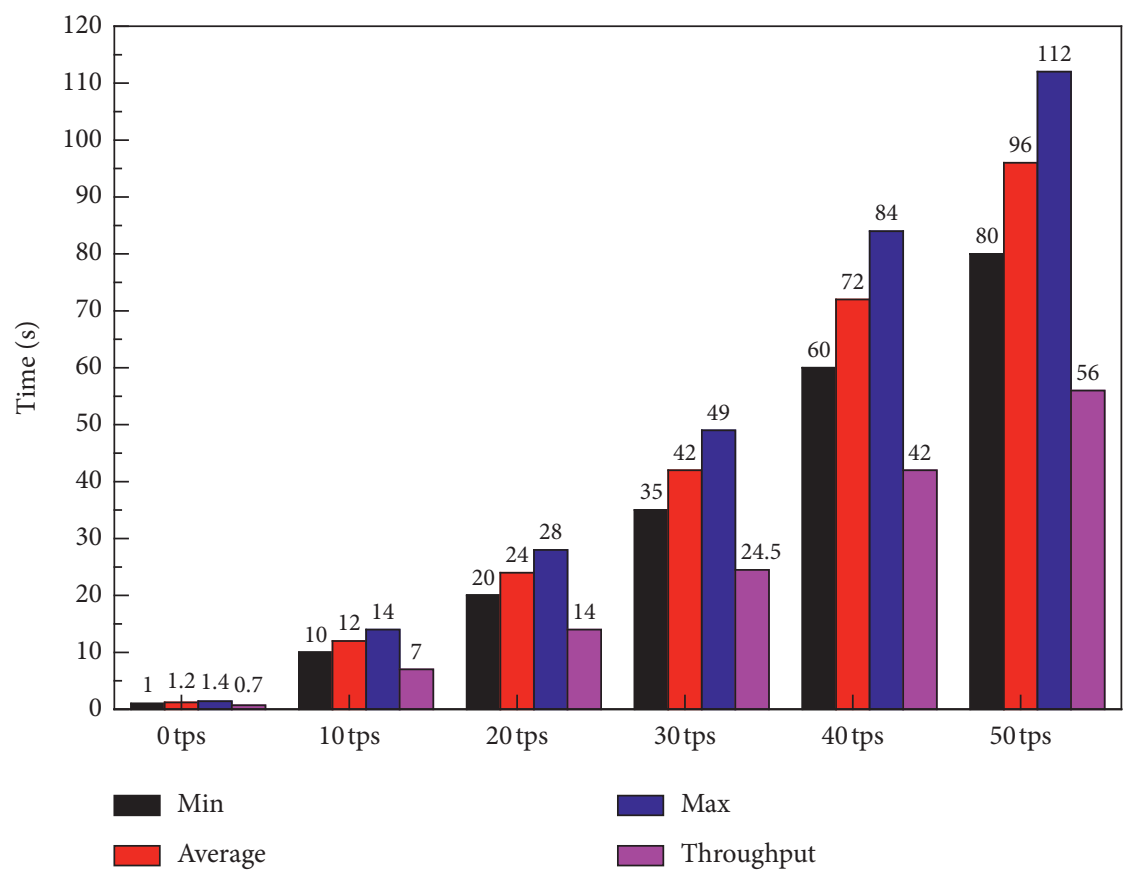

FIgURE 7: The results of performance test.

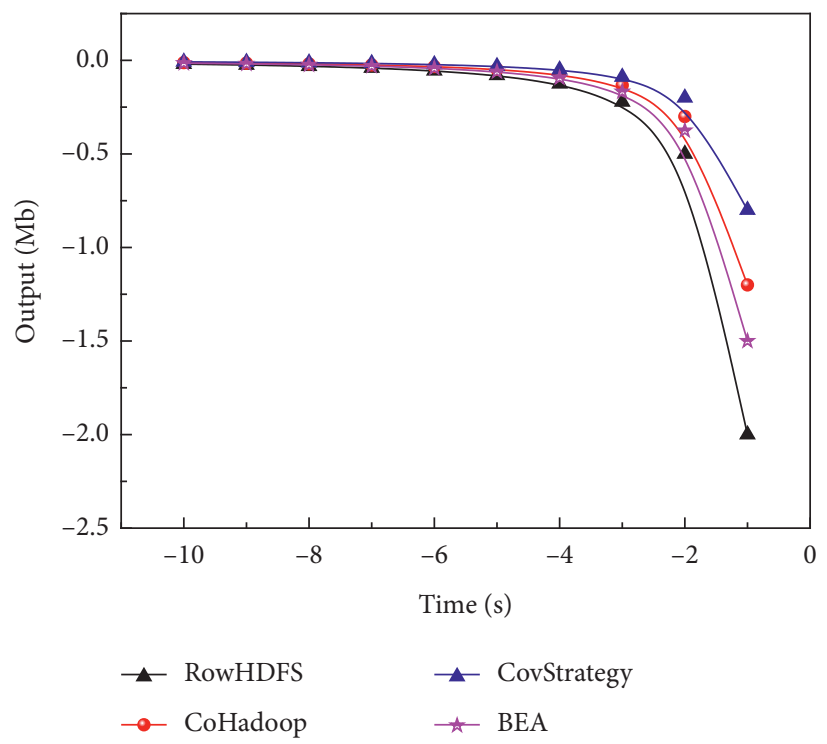

FIgURE 8: Comparison of query performance of two tables' joins under different data distributions.

dataset table assignment; verify the data correlation strategy by comparing the traditional data assignment method and the improved data assignment method in the experiment.

\section{Conclusion}

The concepts, overall framework, and related work related to large-scale transaction data processing in e-commerce are summarized and concluded. First, the business characteristics and several key technologies in the e-commerce domain are briefly introduced. Secondly, the process and main methods of large-scale transaction data processing in e-commerce are introduced, focusing on the application and progress of big data technologies in large-scale task processing. Finally, the current work on task scheduling, data distribution strategy, and data mining and analysis in largescale data processing of e-commerce is summarized. Business models do not rely on traditional cross-border e-commerce platforms. This type of model is likely to emerge in the future for two main reasons, including the following. This type of model will fundamentally impact the traditional e-commerce platform business model. The design of this type of platform business model will be very exclusive to cross-border e-commerce platforms because this type of 
model is designed to redistribute the excess profits earned by traditional e-commerce platforms to other participants. This is bound to harm the interests of traditional e-commerce platforms. This type of model is difficult to be accepted by traditional platforms, which will leave new market opportunities. However, in terms of the entire international trade industry, this is still only a very small part, and there are still more areas where there is room for expansion. To do this, it is necessary to reduce the cost of entry for practitioners in other fields. For example, it was difficult for silk chain financial service providers to enter the market of small and medium-sized enterprises, but by using blockchain technology to reduce the cost of trust, financial service providers can enter the platform to provide services.

\section{Data Availability}

The data used to support the findings of this study are available from the corresponding author upon request.

\section{Conflicts of Interest}

The authors declare that they have no known conflicts of interest or personal relationships that could have appeared to influence the work reported in this paper.

\section{References}

[1] H. Wang, Y. Li, X. Zhao, and F. Yang, "An algorithm based on Markov chain to improve edge cache hit ratio for blockchainenabled IoT," China Communications, vol. 17, no. 9, pp. 66-76, 2020.

[2] E. Ahmed, A. Ahmed, I. Yaqoob et al., "Bringing computation closer toward the user network: is edge computing the solution?" IEEE Communications Magazine, vol. 55, no. 11, pp. 138-144, 2017.

[3] T. Qiu, J. Chi, X. Zhou, Z. Ning, M. Atiquzzaman, and D. O. Wu, "Edge computing in industrial internet of things: architecture, advances and challenges," IEEE Communications Surveys \& Tutorials, vol. 22, no. 4, pp. 2462-2488, 2020.

[4] Z. Zhou, Q. Wu, and X. Chen, "Online orchestration of crossedge service function chaining for cost-efficient edge computing," IEEE Journal on Selected Areas in Communications, vol. 37, no. 8, pp. 1866-1880, 2019.

[5] L. Ale, N. Zhang, H. Wu, D. Chen, and T. Han, "Online proactive caching in mobile edge computing using bidirectional deep recurrent neural network," IEEE Internet of Things Journal, vol. 6, no. 3, pp. 5520-5530, 2019.

[6] Y. Ren, Y. Leng, Y. Leng, Y. Cheng, and J. Wang, "Secure data storage based on blockchain and coding in edge computing," Mathematical Biosciences and Engineering, vol. 16, no. 4, pp. 1874-1892, 2019.

[7] L. Munn, "Staying at the edge of privacy: edge computing and impersonal extraction," Media and Communication, vol. 8, no. 2, pp. 270-279, 2020.

[8] N. Kherraf, H. A. Alameddine, S. Sharafeddine, C. M. Assi, and A. Ghrayeb, "Optimized provisioning of edge computing resources with heterogeneous workload in IoT networks," IEEE Transactions on Network and Service Management, vol. 16, no. 2, pp. 459-474, 2019.

[9] L. Liu, X. Chen, Z. Lu, L. Wang, and X. Wen, "Mobile-edge computing framework with data compression for wireless network in energy internet," Tsinghua Science and Technology, vol. 24, no. 3, pp. 271-280, 2019.

[10] A. Aral and T. Ovatman, "A decentralized replica placement algorithm for edge computing," IEEE Transactions on Network and Service Management, vol. 15, no. 2, pp. 516-529, 2018.

[11] J. Moura and D. Hutchison, "Game theory for multi-access edge computing: survey, use cases, and future trends," IEEE Communications Surveys \& Tutorials, vol. 21, no. 1, pp. 260-288, 2018.

[12] W. Shen, C. Yang, and L. Gao, "Address business crisis caused by COVID-19 with collaborative intelligent manufacturing technologies," IET Collaborative Intelligent Manufacturing, vol. 2, no. 2, pp. 96-99, 2020.

[13] O. Vaughan, "Working on the edge," Nature Electronics, vol. 2, no. 1, pp. 2-3, 2019.

[14] M. A. Rahman, M. S. Hossain, N. A. Alrajeh, and N. Guizani, "B5G and explainable deep learning assisted healthcare vertical at the edge: COVID-I9 perspective," IEEE Network, vol. 34, no. 4, pp. 98-105, 2020.

[15] X. Lin, J. Li, J. Wu, H. Liang, and W. Yang, "Making knowledge tradable in edge-AI enabled IoT: a consortium blockchain-based efficient and incentive approach," IEEE Transactions on Industrial Informatics, vol. 15, no. 12, pp. 6367-6378, 2019.

[16] Y. Zhou, D. Zhang, and N. Xiong, "Post-cloud computing paradigms: a survey and comparison," Tsinghua Science and Technology, vol. 22, no. 6, pp. 714-732, 2017.

[17] Z. Tan, H. Qu, J. Zhao, S. Zhou, and W. Wang, "Uav-aided edge/fog computing in smart iot community for social augmented reality," IEEE Internet of Things Journal, vol. 7, no. 6, pp. 4872-4884, 2020.

[18] A. A. Abdellatif, A. Z. Al-Marridi, A. Mohamed, A. Erbad, C. F. Chiasserini, and A. Refaey, "ssHealth: toward secure, blockchain-enabled healthcare systems," IEEE Network, vol. 34, no. 4, pp. 312-319, 2020.

[19] E. K. Markakis, K. Karras, A. Sideris, G. Alexiou, and E. Pallis, "Computing, caching, and communication at the edge: the cornerstone for building a versatile 5G ecosystem," IEEE Communications Magazine, vol. 55, no. 11, pp. 152-157, 2017.

[20] W. Duan, J. Gu, M. Wen, G. Zhang, Y. Ji, and S. Mumtaz, "Emerging technologies for 5G-IoV networks: applications, trends and opportunities," IEEE Network, vol. 34, no. 5, pp. 283-289, 2020.

[21] C. C. Byers, "Architectural imperatives for fog computing: use cases, requirements, and architectural techniques for fogenabled iot networks," IEEE Communications Magazine, vol. 55, no. 8, pp. 14-20, 2017.

[22] C. Li, Z. Zhang, W. Wei, H.-C. Chao, and X. Liu, "A possible world-based fusion estimation model for uncertain data clustering in WBNs," Sensors, vol. 21, no. 3, p. 875, 2021.

[23] S. Qi, Y. Lu, W. Wei, and X. Chen, "Efficient data access control with fine-grained data protection in cloud-assisted IIoT," IEEE Internet of Things Journal, vol. 8, no. 4, pp. 2886-2899, 2021. 